\title{
Nano-materials as anode electrocatalysts for microbial fuel cells
}

\author{
Weishan $\mathrm{Li}^{1,2 *}$ \\ ${ }^{1}$ School of Chemistry, South China Normal University, Guangzhou 510006, China \\ ${ }^{2}$ National and Local Joint Engineering Research Center of MPTES in High Energy and Safety LIBs, Engineering Research Center of MTEES (Ministry of \\ Education), and Key Lab. of ETESPG(GHEI), South China Normal University, Guangzhou 510006, China
}

\begin{abstract}
Recent progresses made in our laboratory on nano-materials as electrocatalysts for microbial fuel cells are summarized in this review. Compared to the cathode electrocatalysts for oxygen reduction reaction, which are similar to those used for other fuel cells such as proton exchange membrane fuel cells, our interest is mainly focused on the anode electrocatalysts with the aim to accelerate the charge transfer process on anodes. Various nano-materials have been developed in our laboratory as anode electrocatalysts, based on the concept that the charge transfer kinetics between anode and substrates is highly dependent of the bio-compatibility that favors the formation of biofilm on anode, and electrocatalytic activities towards the oxidation reactions of the redox couples in bacteria and the intermediates fermented by bacteria.
\end{abstract}

\section{Introduction}

While rechargeable lithium battery that was invented three decades ago is becoming more and more popular in today's market of electrochemical energy storage and conversion, hydrogen fuel cell, which has an over one hundred years' history and is believed to be the best technology for energy exploitation, has been being under its way to challenge the tough issues faced in the preparation, transportation and storage of hydrogen [1-6] The difficulties presenting in the wide application of hydrogen fuel cell initiate researchers to find out alternatives to hydrogen fuel cell [1-4]. Among the alternative fuel cells that have been proposed, microbial fuel cell (MFC) is most attractive because it can use complicated compounds (substrates) such as waste as fuel to generate power [1].

The principle of MFC is the rearrangement of the digestion process of nutritive organic compounds (substrates) by organisms into a galvanic cell where the oxidation decomposition reaction proceeds on the bacteria-containing anode and the oxygen reduction reaction takes place on cathode [5-10]. Unfortunately, the power output of MFC is far smaller than other fuel cells, which restricts the application of MFC in large scale. The power output of MFC mainly depends on the charge transfer kinetics of anode and cathode and therefore electrocatalysts are necessary for both electrodes. Therefore, designing electrode electrocatalysts is one of the most important topics in the field of fuel cells. Compared to the cathode electrocatalysts for oxygen reduction reaction, which are similar to those used for hydrogen fuel cell and metal/air batteries [1-3] investigations are mainly focused on preparation and performances of the anode electrocatalysts of MFC.

In this review, recent research progresses that have made in our laboratory on the preparation and performances of nano-materials as anode electrocatalysts for MFC are summarized [11]. With the aim to accelerate the charge transfer kinetics on anode, we design the nano-materials based on the concept that the charge transfer kinetics between anode and organic substrates is highly dependent of the bio-compatibility that favors the formation of biofilm on anode, and electrocatalytic activities towards the oxidation reactions of redox couples in bacteria and the intermediates fermented by bacteria. Improved bio-compatibility [12].

The charge transfer between organic substrates and anode in MFC might take place in two paths: directly or indirectly through bacteria. The direct one is that the substrates lose electrons and are oxidized by the oxidants of the redox couples in bacteria [13-18]. The oxidants accept the electrons released from substrates and turn to be reductants that are oxidized and transfer the electrons to the anode. In this path, physical contact is necessary between anode and bacteria, which requires a good bio-compatibility of the anode. That is to say, biofilm should be formed on anode. Therefore, designing an anode that favors the formation of biofilm is one of the efficient strategies to improve the power output of MFC [19-23].

Carbon materials are usually bio-compatible and widely used as anode of MFC, but leave a question, how do the surface properties of carbon materials affect their bio-compatibility, surface morphology or oxygen-containing group? To address this issue, three carbon felt samples were obtained by different treatments, water cleaning (CCF), heating (HCF) and oxidation with ammonium persulfate (ACF), respectively, and their performances as anodes of MFC were compared [1]. It is found that the power output of MFC depends on the morphology

${ }^{\star}$ Correspondence to: Weishan Li, National and Local Joint Engineering Research Center of MPTES in High Energy and Safety LIBs, Engineering Research Center of MTEES (Ministry of Education), and Key Lab. of ETESPG(GHEI), South China Normal University, Guangzhou 510006, China, Email: liwsh@scnu.edu.cn

Key words: nano-material, anode electrocatalyst, microbial fuel cell, biofilm, charge transfer

Received: September 23, 2019; Accepted: October 18, 2019; Published: October 21,2019 
rather than the oxygen-containing group concentration of the carbon felt surface. CCF, HCF and ACF have their surface oxygen-containing groups of $1.52,0.8$ and $0.45 \mathrm{mM} \mathrm{m}^{-2}$ and specific surface areas of 0.33 , 0.65 and $1.19 \mathrm{~m}^{2} \mathrm{~g}^{-1}$, but yield their maximal power densities of 606,858 and $990 \mathrm{~mW} \mathrm{~m}^{-2}$, respectively [24-26]. This investigation suggests that intensive attention should be paid to the design of surface morphology in order to improve power output of MFC.

Accordingly, two kinds of carbon materials with nano-size pore structures have been designed. One is porous carbon with a defined pore size (DPC), which was prepared by using silica spheres as templates and sucrose as carbon precursor [1]. The as-prepared DPC exhibits superior performance as anode material loaded on carbon felt, delivering a power output of $1606 \mathrm{~mW} \mathrm{~m}^{-2}$ in the solution containing $2 \mathrm{~g} / \mathrm{L}$ glucose. This result suggests that suitable pore size in carbon materials for accommodating bactria will facilitate the formation of biofilm $[27,28]$. The other is porous graphite. A porous graphite (PG) was synthesized by thermally decomposing ferrous gluconate, followed by leaching iron [1]. When evaluated on a naked carbon felt (NCF) anode, the resulting PG provides the MFC based on Escherichia coli with a maximum power density of $2.6 \mathrm{~W} \mathrm{~m}^{-2}$. This excellent performance is attributed to the electronically conductive graphite and porous structure that stimulates bacteria to produce their flagella to firmly bond each other.

Besides the morphology of carbon materials, some metal oxides and binders are found to favor the formation of biofilms. An oxygenrich titanium was prepared by a simple heat treatment and used as anode of MFC [1]. It is found that after heat treatment, titanium contains more surface oxygen atoms and thus delivers higher power output as anode of MFC. Compared with pristine titanium, surface oxygen-rich titanium exhibits better bio-compatibility. Polyvinyl alcohol (PVA) was proposed as a new binder to improve power output of microbial fuel cell [1]. The electrochemical performances of the anode using carbon nanotubes as electrocatalyst and PVA as binder were evaluated in an Escherichia coli based MFC, with a comparison of the conventional binder, polytetrafluoroethylene (PTFE). It is found that PVA is more hydrophilic and has stronger interaction with the bacterium membrane than PTFE. Consequently, the anode with PVA as binder facilitates the formation of biofilm and thus exhibits improved electron transfer kinetics between bacteria and anode, compared to the anode using PTFE. The MFC using PVA produces the largest maximum output power, $1.631 \mathrm{~W} \cdot \mathrm{m}^{-2}$, which is $97.9 \%$ greater than the largest one produced by the MFC using PTFE $(0.824$ $\mathrm{W} \cdot \mathrm{m}^{-2}$ ). The output power of MFC can be further improved by using poly (bisphenol A-co-epichorohydrin) (PBE) as the anode binder [1]. It is found that the MFC using PBE reaches a maximum power density output of $3.8 \mathrm{~W} \mathrm{~m}^{-2}$, which is larger than the MFC using PVA. This improvement is ascribed to the stronger interaction of PBE with bacteria than PVA, which benefits the formation of biofilm.

\section{Improved electrocatalytic activity}

Anode electrocatalysts should possess electrocatalytic activities towards the oxidation reactions of the redox couples in bacteria or the intermediates fermented by bacteria. For the oxidation reactions of organic compounds, platinum exhibits the best electrocatalytic activity [1]. However, platinum is too expensive to be used in MFC. Fortunately, the carbides or oxides of some transition metals possess similar electrocatalytic activity as platinum. Accordingly, various nano-carbides or nano-oxdies have been developed for anode electrocatalyst use.
A pure $\beta$-molybdenum carbide $\left(\mathrm{Mo}_{2} \mathrm{C}\right)$ was prepared by solution derived precursor and used as anode electrocatalyst of MFC based on $K$. pneumoniae. It is found that the maximum power density of single-cube MFC with $6.0 \mathrm{mg} / \mathrm{cm}^{2} \mathrm{Mo}_{2} \mathrm{C}$ loading reaches $2.39 \mathrm{~W} / \mathrm{m}^{3}$, which is comparable to that of the MFC using $0.5 \mathrm{mg} / \mathrm{cm}^{2} \mathrm{Pt}(3.64$ $\mathrm{W} / \mathrm{m}^{3}$ ). With this encouraging result, the the performances of $\mathrm{Mo}_{2} \mathrm{C}$ were further improved by introducing the secondary components. Firstly, metal nickel was introduced. The MFC using $\mathrm{Ni} / \beta-\mathrm{Mo}_{2} \mathrm{C}$ as anode electrocatalyst delivers a higher power density than the MFC using $\beta-\mathrm{Mo}_{2} \mathrm{C}$ as anode electrocatalyst. Then, carbon nano-tubes were introduced. The $\mathrm{Mo}_{2} \mathrm{C} / \mathrm{CNT}$ s composite was synthesized by using microwave-assisted method with $\mathrm{Mo}(\mathrm{CO})_{6}$ as a single source precursor and its electrocatalytic activity was evaluated in the MFC based on Escherichia coli. It is found that the carbon felt electrode with 16.7 wt \% Mo loading exhibits a comparable electrocatalytic activity to that with $20 \mathrm{wt} \%$ platinum as anode electrocatalyst. The superior performance of the developed platinum-free electrode can be ascribed to the bifunctional electrocatalysis of $\mathrm{Mo}_{2} \mathrm{C} / \mathrm{CNTs}$ for the conversion of organic substrates into electricity through bacteria. The composite facilitates the formation of biofilm, which is necessary for the electron transfer via c-type cytochrome and nanowires. On the other hand, the composite exhibits the electrocatalytic activity towards the oxidation of hydrogen, which is the common intermediate fermed by bacteria. It is also found that $\mathrm{MoO}_{2}$ can improve the electrocatlytic activity toward the oxidation of the intermediates fermented by bacteria [4].

A composite, polyaniline (PANI)/mesoporous tungsten trioxide $\left(\mathrm{m}-\mathrm{WO}_{3}\right)$, was developed as a platinum-free and biocompatible anodic electrocatalyst of MFC. The $\mathrm{m}-\mathrm{WO}_{3}$ was synthesized by a replicating route and PANI was loaded on the $\mathrm{m}-\mathrm{WO}_{3}$ through the chemical oxidation of aniline [4]. It is found that the composite exhibits a unique electrocatalytic activity, which can be ascribed to the combination of $\mathrm{m}-\mathrm{WO}_{3}$ and PANI. The $\mathrm{m}-\mathrm{WO}_{3}$ has good biocompatibility and PANI has good electrical conductivity. Most importantly, the combination of $\mathrm{m}-\mathrm{WO}_{3}$ and PANI improves the electrochemical activity of PANI for proton insertion and de-insertion, which are beneficial for the oxidation of the intermediates fermented by bacteria.

The nano-materials that exhibit electrocatalytic activity for mixed bacterium culture rather than a single bacterium were also considered. Iron carbide nanoparticles dispersed in porous graphitized carbon (Nano-Fe $\mathrm{N}_{3} @ \mathrm{PGC}$ ) was synthesized by a direct pyrolysis of ferrous gluconate and a following removal of free iron and used as anode electrocatalyst [4]. Equipped with Nano-Fe ${ }_{3} \mathrm{OPGC}$, the MFC based on a mixed bacterium culture yields a power density of $1856 \mathrm{~mW} \mathrm{~m}^{-2}$. This excellent performance is attributed to the large electrochemical active area and the high electronic conductivity that porous graphitized carbon provides and the enriched electrochemically active microorganisms and the enhanced activity towards the redox reactions in organisms by $\mathrm{Fe}_{3} \mathrm{C}$ nanoparticles.

Cobalt-modified molybdenum dioxide nanoparticles highly dispersed on nitrogen-doped carbon nanorods $\left(\mathrm{Co}-\mathrm{MoO}_{2} / \mathrm{NCND}\right)$ was also developed as anode electrocatalyst for mixed bacterium culture. Molybdenum dioxide nanoparticles dispersed on carbon nanorods $\left(\mathrm{MoO}_{2} / \mathrm{CND}\right)$ are first synthesized by annealing $\mathrm{Mo}_{3} \mathrm{O}_{10}\left(\mathrm{C}_{6} \mathrm{H}_{8} \mathrm{~N}\right)_{2} \cdot 2 \mathrm{H}_{2} \mathrm{O}$ nanorods under a control of argon flow rate, and the $\mathrm{Co}-\mathrm{MoO}_{2} / \mathrm{NCND}$ is obtained by a hydrothermal reaction of $\mathrm{MoO}_{2} / \mathrm{CND}$ with cobalt acetylacetonate and the subsequent $\mathrm{N}$-doping by dicyanamide. Physical characterizations indicate that the as-synthesized $\mathrm{Co}-\mathrm{MoO}_{2} / \mathrm{NCND}$ is composed of molybdenum dioxide nanoparticles modified with cobalt oxide and highly dispersed in nitrogen-doped carbon nanorods that 
inherit the precursor morphology. The electrochemical measurements demonstrate that the MFC using this anode electrocatalyst in phosphate buffer solution containing $1 \mathrm{~g} \mathrm{~L}^{-1}$ sodium acetate delivers a maximum power density of $2.06 \pm 0.05 \mathrm{~W} \mathrm{~m}^{-2}$, which is strikingly higher than the bare carbon felt anode $\left(0.49 \pm 0.04 \mathrm{~W} \mathrm{~m}^{-2}\right)$. This improved performance is ascribed to the large surface area and the electronically conductive path that nitrogen-carbon nanorods provide and the enhanced electocatalytic activity of molybdenum dioxide by cobalt modification toward redox reactions in bacteria.

\section{Conclusion}

The power out of microbial fuel cells is mainly restricted by the sluggish charge transfer kinetics on anode and cathode. On the anode, the charge transfer kinetics is highly dependent of the bio-compatibility and the electrocatalytic activity of the anode. The bio-compatibility of the anode can be improved by delicately designing surface morphology of carbon materials, especially their pore structure, introducing some metal oxides such as titanium oxide, and using the binders that exhibits strong interaction with bacteria. On the other hand, some transition metal carbides or oxides such as molybdenum, tungsten and iron carbides exhibit electrocatalytic activity towards the oxidation reactions of redox couples in bacteria and the intermediates fermented by bacteria, which is comparable to metal platinum. The performances of these materials can be further improved when they are used in highly dispersed nanoparticles.

\section{References}

1. Manoharan Y (2019) Hydrogen fuel cell vehicles; current status and future prospect, applied. Science 9: 2296

2. Wen YC (2019) Covalent organic framework-regulated ionic transportation for highperformance lithium-ion batteries. Journal of Materials Chemistry A 7: 26540-26548.

3. Guo RD (2019) Tailoring low-temperature performance of a lithium-ion battery via rational designing interphase on an anode. ACS Appl Mater Interfaces 11: 3828538293 .

4. Lin $\mathrm{ZH}$ (2019) Insights into the interfacial instability between carbon-coated $\mathrm{SiO}$ anode and electrolyte in lithium-ion batteries. J Phys Chem C 123: 12902-12909.

5. Liao B (2018) Designing low impedance interface films simultaneously on anode and cathode for high energy batteries. Adv Energy Mater 8: 1800802.

6. Chen M (2017) Morphology-conserved transformations of metal-based precursors to hierarchically porous micro/nanostructures for electrochemical energy conversion and storage. Advanced Materials 29: 1607015.

7. Stephens EL (2016) Toward sustainable fuel cells. Science 354: 1378-1379.

8. Wang XJ (2014) Pore-arrayed hydrogen molybdenum bronze: Preparation and performance as support of platinum nanoparticles for methanol oxidation. $J$ Power Sources 259: 255-261.
9. Fu Z (2012) Mesoporous tungsten carbide-supported platinum as carbon monoxidetolerant electrocatalyst for methanol oxidation. Int J Hydrogen Energy 37: 4704-4709.

10. Li WS (2005) Chemicals and energy co-generation from direct hydrocarbons/oxygen proton exchange membrane fuel cell. J Power Sources 145: 376-382.

11. Zhang LX (2008) Microbial fuel cell based on Klebsiella pneumoniae biofilm. Electrochem Commun 10: 1641-1643.

12. Zhong YT (2019) Hierarchical Co3O4 nano-micro arrays featuring superior activity as cathode in flexible and rechargeable zinc-air battery. Advanced Science 6: 1802243.

13. Huang YJ (2012) Manganese dioxide with high specific surface area for alkaline battery. Chem. Res. Chinese University 28: 874-877.

14. Huang YJ (2013) Controllable syntheses of $\alpha$ - and $\delta-\mathrm{MnO} 2$ as cathode catalysts for zinc-air battery. Electrochim Acta 99: 161-165.

15. Cui D (2014) Which determines power generation of microbial fuel cell based on carbon anode, surface morphology or oxygen-containing group? Int J Hydrogen Energy 39: 15081-15087.

16. Chen XF (2015) Porous carbon with defined pore size as anode of microbial fuel cell Biosens Bioelectron 69: 135-141.

17. Xiong J (2018) Porous graphite: a facile synthesis from ferrous gluconate and excellent performance as anode electrocatalyst of microbial fuel cell. Biosensors and Bioelectronics 109: 116-122.

18. Zhou XW (2016) Surface oxygen-rich titanium as anode for high performance microbial fuel cell. Electrochim Acta 209: 582-590.

19. Chen XF (2015) Improved power output by incorporating polyvinyl alcohol into the anode of a microbial fuel cell. J Mater Chem A 3: 19402-19409.

20. Li HY (2018) Power output of microbial fuel cell emphasizing interaction of anodic binder with bacteria. J Power Sources 379: 115-122.

21. Fu Z (2010) Preparation and activity of carbon-supported porous platinum as electrocatalyst for methanol oxidation. Int J Hydrogen Energy 35: 8101-8105.

22. Zeng LZ (2010) Molybdenum carbide as anodic catalyst for microbial fuel cell based on Klebsiella Pneumoniae. Biosens Bioelectron 25: 2696-2700.

23. Zeng LZ (2012) Ni/ $\beta-M o 2 C$ as noble-metal-free anodic electrocatalyst of microbial fuel cell based on Klebsiella pneumoniae. Int J Hydrogen Energy 37: 4590-4596.

24. Wang YQ (2014) Nano-molybdenum carbide/carbon nanotubes composite as bifunctional anode catalyst for high-performance Escherichia coli-based microbial fuel cell. Biosens Bioelectron 51: 349-355.

25. Zeng LZ (2018) Highly dispersed polydopamine-modified Mo2C/MoO2 nanoparticles as anode electrocatalyst for microbial fuel cells. Electrochem Acta 283: 528-537.

26. Wang YQ (2013) Polyaniline/Mesoporous tungsten trioxide composite as anode electrocatalyst for high-performance microbial fuel cells. Biosens Bioelectron 41: 582 588

27. Hu MH (2019) Nano-Fe3C@PGC as a novel low-cost anode electrocatalyst for superior performance microbial fuel cells. Biosensors and Bioelectronics 142: 111594.

28. Li X (2019) Co-modified MoO2 nanoparticles highly dispersed on N-doped carbon nanorods as anode electrocatalyst of microbial fuel cells. Biosensors and Bioelectronics 145: 111727.

Copyright: (C2019 Weishan Li. This is an open-access article distributed under the terms of the Creative Commons Attribution License, which permits unrestricted use, distribution, and reproduction in any medium, provided the original author and source are credited. 